\title{
HOW TO MAKE CLASSROOMS CREATIVE AND OPEN SPACES. ARIS GAMES, DIGITAL ARTIFACTS AND STORYTELLING
}

\author{
Fred Adam; Mauricio O'Brien; Arianna Mazzeo; Veronica Perales \\ Elisava Universitat Pompeu Fabra, Universitat de Murcia \\ fredadam@um.es, mobrian@elisava.net, amazzeo@elisava.net, \\ vperales@um.es
}

\begin{abstract}
As part of long-term research into interviewing users and visualizing digital artifacts, we have created parallel archives of projects in our classroom. Ethnography helps us to discover the temporal trends of interactions with students and with the virtual environment.

The outcomes expected motived us to repurpouse stories we co-create with students in a new form, retelling motivations, design, narratives, into a gaming scenario where the use of experiences become more digital and less tangible but always snapshots of their social existence.
\end{abstract}

Keywords: Innovation, creativity, design ethnography, trans-media, storytelling, teaching methods. 


\section{INTRODUCTION}

Design and Cultures brings together key thinkers and practitioners involved in making and theorizing our contemporary material and immaterial world: its ritual, its aesthetics, behaviours and interactions.

The creative classroom projects we develop at Elisava University Pompeu Fabra into the Master in Design and Architecture and at University of Murcia, Faculty of Art are based both on interactive workshops to pretend and make a shift in the way users conceptualize, envisage, engage in object culture by a personal mobile journey. Designers, learners and teachers as facilitator are now invite to map their personal environment, using a mobile phone, a tablet or a smart device as digital artifact as likely to engage in social science and research techniques.

Once on intuitive process, gauging cultural relevance of the context the multiple pathways they suppose to live, users involved become part of a burgeoning area, design ethnography. Human focus on everyday practices, (storytelling, gamification, augmented reality and a wiki space) as common working scenario, are essential tools in interpreting the complex implications of culture as prosumer of globalized values, in a social understanding of 21st century interplay of our technologically mediated culture and designer's observation.

\subsection{M-Learning Workshop in Máster en Diseño y Arquitectura en Elisava, Escuela Superior de Diseño e Ingeniería.}

In recent years, ethnography has become popular with designers of products and technologies as a way of learning about the experience of the users. This research approach has been applied to such diverse problems as:

- How to design new environments that encourage group work and collaboration -How to design m-learning framework that fit the "mental model" and usage patterns of their target audiences

- How to design events, services or products exhibits that maximize the engagement between visitors and devices, in a social learning environment.

Design ethnography is a rapidly growing field of employment for those who wish to work in applied anthropology and social sciences. In this course, students learn the fundamentals of the field. By collaborating on an applied project, they gain practice in applied research methods and video ethnography. They learn to engage in collaborative analysis, and they work with users, to translate their research into practical applications. In addition to the hands-on experience, students conduct readings on topics relevant to the project and to design anthropology, social sciences in general. This 4- classes project will be an exploratory research study on environmentally friendly design for m-Learning using m-phones to promote a music festival.

\section{Course Requirements}

\section{Teamwork and participation (20\%)}

One of the key skills of a practitioner in applied ethnography and is the ability to work well as part of a team. In this class, you will be evaluated on your teamwork skills, including:

- Active and thoughtful participation in class discussions, both about class reading sand about the class project

- Staying focused on the client's needs

- Good communication and collaboration with your fieldwork partner

- Equal distribution of work between you and your field work partner 
- Carrying out your responsibilities in a timely manner

- Good communication with the team leader/professor.

\section{Interviews (15\% x 2)}

Instructions for each will be handed out on the first day of class. Students provide to bring their own pc and camera recording.

\section{Class project (50\%)}

The project consists of a series of activities. You will receive agrade for completing each step.

\section{Project Information}

\section{Project Title}

LOTTAROX: Exploratory Research on an Interactive Music Festival

\section{The Client}

Our client organization is Lottarox Agency. We will work with Marzio, Ceo of Lottarox London based.

\section{Overview of Purpose}

This project is exploratory in nature. It is designed to elicit new ideas for intercation by design and friendly Lottarox products, ideas that will appeal to the hearts and minds of "music" consumers. The study our class will conduct investigates what motivates people to purchase "music" products (apps), the relationship between features of "music" products and consumer selfrepresentation, and what makes a product or a service in the eyes of the consumer. Are consumers purchasing "music" products/services because they are environmentally conscious, health conscious, aesthetically conscious, or some combination of these and other kinds of sensibilities? How do they use "music" products/services to represent themselves to others? What makes people purchase an App? Answers to these questions can then be used to brainstorm innovations in the design and development of Lottarox products/Services. The research question:

- What makes people interested in purchasing and using friendly music products/services?

- Assumptions: People use the consumption of "music" products to represent themselves to others (a form of presentation of self).

- There are a certain number of identifiable core attributes possessed by "music" Products/services that work to entice consumers/users. People are members of communities that share values such as the environment and social behaviours; they display their membership in such communities in various ways.

\section{The Research Process}

A. Recruit Participants

B. Data Collection

Data collection will consist of two parts. The first part will be giving study participants a disposable camera and instructions to take pictures of all the products in their homes that they consider "music". Participants will be given a day to engage in this photo- taking activity and inteviews by their own camera. The researchers will then have the photos developed and prepare for the semistructured interviews. The second part of data collection will be in-depth interviews with the community/participants. It is anticipated that these interviews 
will be $11 / 2$ to 2 hours in length. They will be video and audio taped for analysis purposes. Students will conduct fieldwork in pairs or a group of 3-4 people.

\section{Data Analysis}

Much of the analysis process will be done in class, as a group. Data analysis will follow a trajectory of identifying instances, patterns, and models. Instances are "events, behaviors, statements, or activities that stand out because they occur often, because they are crucial to other items, because they are rare and influential, or because they are totally absent despite the researchers' expectations" (LeCompte and Schensul, p.150). Patterns are based on groups of items that fit together, express a particular theme, or constitute a predictable and consistent set of behaviors. Models are frameworks for understanding the data in a way that answers the client' questions; they draw on patterns and integrate them with theory.

\section{Report}

In day 4, students will develop a list of topics to cover in the client report. Each student will write the written report. They will analyze everyone's field notes and videoclips to identify overall patterns regarding the topic of the global video. Students will also present the information whit the final client presentation.

\section{Client Meetings. In video conference}

Prepare the designer of the future for the development, creation and communication of contemporary architecture and design in areas that should be addressed from a multidisciplinary perspective and open.

- Mastering the parameters that make up a communication, increasingly complex, global and multiplatform. Instruction in practical tools to structure an iterative project (design thinking);

- Experience the interdisciplinary approach to design and architecture in all its forms, and to promote innovation in new areas of dissemination of information and knowledge;

- Provide communication skills and expand knowledge on the complexity of the different phases media broadcast transmedia;

- Sustain a real project organized around the definition of conceptual strategies, their development and their appropriate architecture applied in different communication media.

The practical application lies in a workshop where you add in stockings architectural projects mentioned above as transmedia, gamification technique, the average geolocative and augmented reality.

The architecture transmedia help boost iteration channels and interaction of users with the inclusion of "artifacts" that allow technology as in the results presented, CabLab (Anna Naomi and Eva Yubero Amano) and Reflexions (Daniela Fullenkamp and Ernesto Morales). Both approach their interventions since the creation of sensitive to human interaction that extends the perception of the media and mentioned as Jared Schiffman (Interview) "All media occupy physical space. Books and magazines on paper, films and television on screens, signs and advertisements on posts and billboards. Not all media, however I, are interactive. With the advent of the computational systems, the development of large-scale display devices, and recent improvements in sensing technologies, the landscape of interactive media fundamentally has changed. "

The strategic factor in the proposed Gamification Get-a-life (Rebeca Fernandez and Nico scrambled) and WTF (Fred Cruz) architecture that use real as videogame scenario thereby contextualizing the user in an interactive space to enjoy in a playful way. In both cases plantaean user immersion in architecture from the key role that video games encourage real collaboration between users 
and which is similar to collaborative learning environments and collaborative work environments in which participants share information and learn of others (collaborative work environments assisted by computer, for example). Multiplayer Video games develop both competitiveness and collaboration, encourage players to join teams (or clans) and compete against other teams. For example, role play massively multiplayer online (MMORPG) that was based Get-a-Life, players can create teams, share information via voice or text and learn by watching other players in different scenarios and missions within Parc del Forum in Barcelona through tablets.

In the case of Geolocative Media on which sits the proposal for the Film Library of Catalunya, Reflexions (Ignacio Cardenas) opens a work environment where audiovisual content of the institution are implemented in the city of Barcelona. It expands the presence of the Film in the city and freed from the physical limitations to consider fascinating aspects of the latest communication technologies geolocation, which act between the virtual and the real and digital worlds transcend in local. These technologies have opened new social within social networking sites and gaming platforms worldwide, allowing users to browse both physical and virtual sites simultaneously. This phenomenon is made possible by the fact that aggravates the mobile technology such as mobile phones, it has become common routine, and the ability of users to access SMS, MMS, MP3, email, camera functions and free Internet allow users to experience the place and spatiality as never before (Chan, D 2008).

\section{HOW ARIS GAMES OPEN NEW CREATIVE PATHWAYS. THE CASE OF UNIVERSITY OF MURCIA, FACULTY OF ART}

Digital technologies of information and communication are integrated, increasingly, in schools. The smartphone is your space in a very clear role: articulates knowledge and physical space, the Mobile Learning is the "knowing" in place. The game from Pocket microcomputers is a strategy to attract students to a learning process. Teaching from the Fine Arts, which includes courses audiovisual production and editing, as well as studies on interactivity, enables the Mobile Learning deal from a position of excellence. Our approach has the background knowledge to audiovisual and artistic criticism, linked as we will see, among others, to film and documentary (mainly experimental).

We work in class with emerging modes of creation, as is the case with the transmedia, this allows us to combine the conception and design (objects, scenes, actors ...) analog with physical and virtual public space. Our challenge is to develop teaching strategies that allow vehicular projects where there is a transfer of technical and theoretical resources, training students to be competitive in this profession. ARIS Games is a marvelous tool for this purpose, allows us to experience and write in the key of audiovisual XXI century, in the creative ways that respond to the social and technological changes of our time.

\subsection{The Smartphone: microcomputer in the classroom}

From the field of education is much talk about the possibilities and potential of learning supported by digital tools. For the past several years, both as teachers and students use computers to complete the knowledge gained in classes and courses, most of the centers are equipped with computer rooms connected to the Internet. The entry-recent and still very limited-pocket microcomputers (smartphones, tablets, iPads...) is causing the birth of a new type of learning 
content adapted to these novel mobile media. We then speak of a "learning on the move '.

We could say that mobile phones are perceived classroom-so far and at least in Europe, as a distraction for students. Introduce social networks within the walls of the school and these networks are translated as antithetical to the aims pursued by teachers. The runaway smartphone act as disruptive in the learning cycle of students. It is true that the vast majority of teenagers use their mobile devices to chat with friends, see the relevance of his social network, or simply gossip. This type of activity, which can also become a compulsive habit starts to emerge with a strong applications of distraction and little interest from the point of view of social involvement documented and conscious. Take the example applications like Gossip (http://www.gossip.io) used at times to exercise cyberbullying. The concept of learning from your mobile or smartphone is then, a priori, a vague idea that does not seem to take shape in the imaginary teacher, at least as true teaching tool. And yet a significant proportion of young students in their pockets these devices abundantly computing power than the computers of a decade ago. This paradoxical encounter between the potential and the use of these mobile devices can swap if, from schools, arise new forms of use, suitable for both "reading" to "writing" with objectives related to training.

To truly understand why and how mobile technologies advanced telecommunications can have a positive impact on the education system, we evaluate two advantages inherently entail. The first is simple, consisting of interest from geographic context digital information. It seems obvious, but until recently, information reached us through a stationary screen, often static in one place. The information was spatially disconnected at all times (despite geographical references that we could get through that screen in question...). Access to audiovisual content in specific locations most sense when there comes an intense dialogue between space and information, when the spatial location is not only relevant in the interpretation of information, but the experience is constitutive of knowledge. It is at this point that the use of geolocation technologies and associated applications allow us, on the one hand complex design learning experiences that operate with axes of time, space and information, on the other, to experience such creations. This entire framework enriches our reading of our environment, situated in the actual context relevant information, greatly facilitating the assimilation of it as information becomes 'experienced', not just learned (what we call "learning by heart ").

The second advantage lies in the fact that all this information received insitu not operate unidirectionally, where the receiver is only reader. One of the most significant changes in these new cultural variants is the transformation of lectoautor reader, the advice in the book Isidro Moreno speeches Creativity and hypermedia, "The lectoautor claims a degree of ownership and increasing autonomy (...) The topical phrase, "Now the work is the reader" becomes reality. Any work on digital media is open and manipulable and, to some extent, becomes hypermedia, although it has been designed with linear thinking "(Moreno, 2012: 39). Hypermedia and transmedia creations a priori this nature would "open" passive reading surpassed the means to address active experiences, dynamic, dialogic. These new forms of writing should raise the involvement in this process of digital literacy in the real context. The younger generation would have to be, and no doubt will be, participants in this description of society who make. 
The merger between reading the information attached to the place and the opportunity to participate in their own redefinition is promising framework for a new way of teaching that transcends the knowledge beyond the physical boundaries of the school to be lived, understood and enhanced if be, in the public space. American Professor Kevin J. Pugh says about "Just as experience is a means for enriching and expanding learning, so learning is a means for enriching and expanding experience".

The Mobile Learning is an exceptional opportunity to guide students toward new behavior-supported-education regarding the use of their smartphones, making them see the enormous potential of these devices. It is also a key to end the educational space partitioning and public space, creating versions that hybridize both dimensions. Communication technologies allow us to truly deal with learning processes in fusion with everyday life anywhere. Our environment is extremely rich, there is no better support than life to discover and understand the phenomena associated with it. Strengthening the link between knowing and living, or what is the same theory and practice, formula and application ... may be one of the most important achievements of this wave of Mobile Learning.

\subsection{ARIS Games like authoring environment}

In this framework, ARIS Games is one of the few environments interactive digital content creation geolocated, designed as a teaching tool and allows exploring these new forms of writing without computer skills. This tool consists of an online editor accessible from a web browser that allows you to position a set of media (images, text, audio, video ...) on a world map. Access to this content is carried out through a client application for iPhone, iPad and iPod propose allowing application prototypes Mobile Learning from optimal conditions. Games ARIS project was born in 2010 at the University of Wisconsin, Madison (U.S.). It is run by David Gagnon that has the support of many faculty and fellows of his college students and teachers in collaboration with other universities as Chris Holden professor at the University of New Mexico. We, Veronica Perales and Fred Adam, participated as international partners from the Faculty of Fine Arts in Spain. During the 2012 work on the translation of the client application for smartphone fracas and Spanish, and made some video tutorials on using the tool in these languages (these tutorials are accessible from the website Hypermedia Creation Lab and Transmedia within the University of Murcia). The lab (Fig. 1) is linked to GPS Museum which operates as examples source window and addressing the practical application of concepts such as hypermedia, transmedia or expanded cinema.

Our goal in what follows is the presentation of the use of ARIS Games in our courses at the Faculty of Fine Arts at the University of Murcia, as a support tool when implementing new forms of narrative and nonlinear geolocated interactively. Our courses audiovisual address different domains that gather around the concepts of hypermedia, Transmedia and Expanded Cinema (including the film). In a very synthetic describe the aspects that differentiate these three lines of development, we will develop later. Hypermedia includes interactive audiovisual that can be modified by users, endowed with a certain autonomy and mutability, the transmedia is differentiated by combining analog and digital media in the narrative, each piece works as part of a puzzle that is the 'image' overall, is expanded cinema experimental and documentary film language outside the boundaries of traditional cinema. As we can see already from this first introduction to the terms, it is not watertight compartments, but opens up a huge 
range of possibilities, combinatorial and miscellaneous. On the website of Creation Lab Transmedia Hypermedia and you can see examples of projects developed in class and other information related to pedagogy partially revealing our working methods.

\subsubsection{Multiple different contexts Practices}

In April 2011, we participated in the ARIS Game Jam Games in three days building the prototype of an application called Microcosmos Explorer Aris which is the first year the team within the University of Murcia. From this first positive experience (both in the process and outcomes) started in 2011 as a tool ARIS include possible within some classes of audiovisual content and to students in their final year undergraduate and / or degree in Fine Arts. After three years of research and use of ARIS we have the depth of field needed to make a general assessment of the pedagogical experience.

In the summer of 2012 during a trip to the United States, and after two years of distance collaboration over the Internet, we had the opportunity to meet much of the ARIS team in Madison. It was a very enriching in which clearly understood the magnitude and variety of possible uses of the application. The approach from the team at the University of Murcia are giving is fundamentally pedagogical tool, closely related to the idea of Mobile Learning. However, the development team is far from taking this line as one of its premises, its main interest is fun, playful evidently understood as a very serious matter. This leads us to consider questions such as the link between play and learning all about gamification analyzes that place the importance of fun in the process of assimilation of knowledge. Educational and recreational applications Applications are closer to what we wanted to see for a long time.

From the Lab, we chose to ARIS as an exceptional tool for creating interactive audiovisual geolocated, without emphasizing its "primary root" that would be playful. This decision comes our desire not to condition the design of projects within courses, well that many of them have a marked game. However, the terminology used in the context of the application is undoubtedly entertaining, each application is a game or called game, there are "challenges" are "players"... interface elements have implicit this brand developers consciously assigned. But students quickly understand when we boarded the editor, that the structure of a game is a potential area interactive, dialogic, dynamic, we can locate the chosen content. The contents and designate bring character to the application, determining its classification in the broad cultural spectrum. The game, and we agree fully with the ARIS team in Madison, is a component and extremely powerful strategy, can seduce and engage the public. The gaming experience is not to be necessarily a break superficial for years know the line of serious games (Serious Games), which would be how issues "serious" from the playful. The game should not be regarded as a trivialization of content, the game is a key. It is also important to know the profile of the audience you are targeting the games, in the U.S. there are a good number of teachers working with young students (equivalent to what in Spain would ESO studies) through applications made with ARIS. Logically, the contents must be structured in order to maturity of recipients. Make serious games for children is not the same as adults do, nor is it comparable type of game (challenges, items...), but in both cases the game is a strategy, a vehicle to raise difficult issues. 
The use of mobile terminals is essential in these applications, no matter how fun the same. However, from our knowledge we find that in 2013, the vast majority of European Arts schools have no materials that include the development of audiovisual content on mobile phones and tablets. This should make us reflect on the integration of smartphones late as visual support in public higher education institutions in Europe and perhaps in the impact it has when considering this type of device as a distraction in the classroom, rather than as a resource. If the game, as a means, is a motivating argument in the education of young students in the United States, the need for specialists who develop these applications is evidence playful rotunda. Behold the interest of educating students of art that can cover this area of creation, which undoubtedly occupy an important place in the cultural industries near future.

\section{Aris 2.2.2 Microcosm Explorer, an application prototyped}

Our first prototype space game with him titled ARIS Aris Microcosm Games Explorer (Fig. 1).

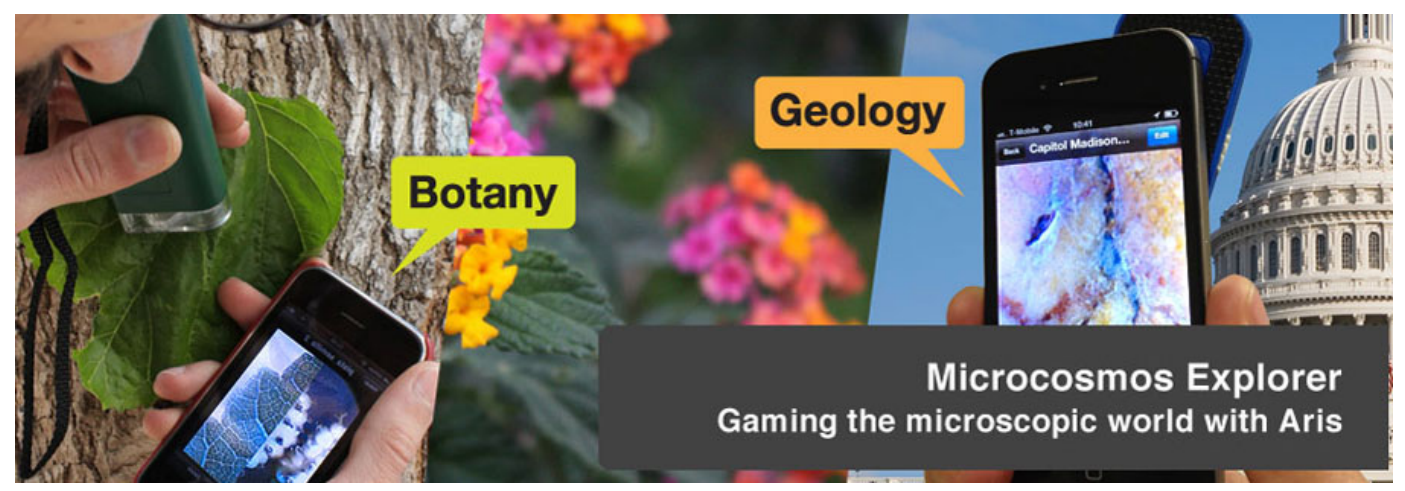

Fig.1.

It is a mobile learning application for learning in the area of Botany. We take a complex of themed gardens is located in the field of Espinardo School of Murcia and present content following a set of clues. The narrative guidance is given by a fictional character based on a historical figure of the nineteenth century, a botanist named Gaston Bonnier which itself is present in the Museum Lousteau, also within Espinardo. The main mechanism of the game is based on the search of seeds and planting Gaston lost-virtually-in the appropriate places along the characteristics of the gardens. Players can see on their screens different views or plant macro photographic images, must find, using a portable microscope for cellKeeploop - the place to plant. They must know and recognize the leaves and / or flowers of plants. The goals achieved are various, firstly the Biology and related studies, are a fun exercise that tests their knowledge of botany. The exploration of the microcosm is possible and indeed desirable for the proper understanding of our environment. On the other hand, it creates a collaboration between two different degrees (at least two) within the university, which is a manifestation of the desired feaciente mainstreaming. Creating Mobile Learning content is a good opportunity to generate cross-disciplinary collaborations between art studies and other races and favors the emergence of innovative pedagogical practices. The smartphones-in this case equipped with these optical attachments become affordable-sensory augmentation agents, implementing our perception of the environment, stimulating our senses (Fig. 2). 

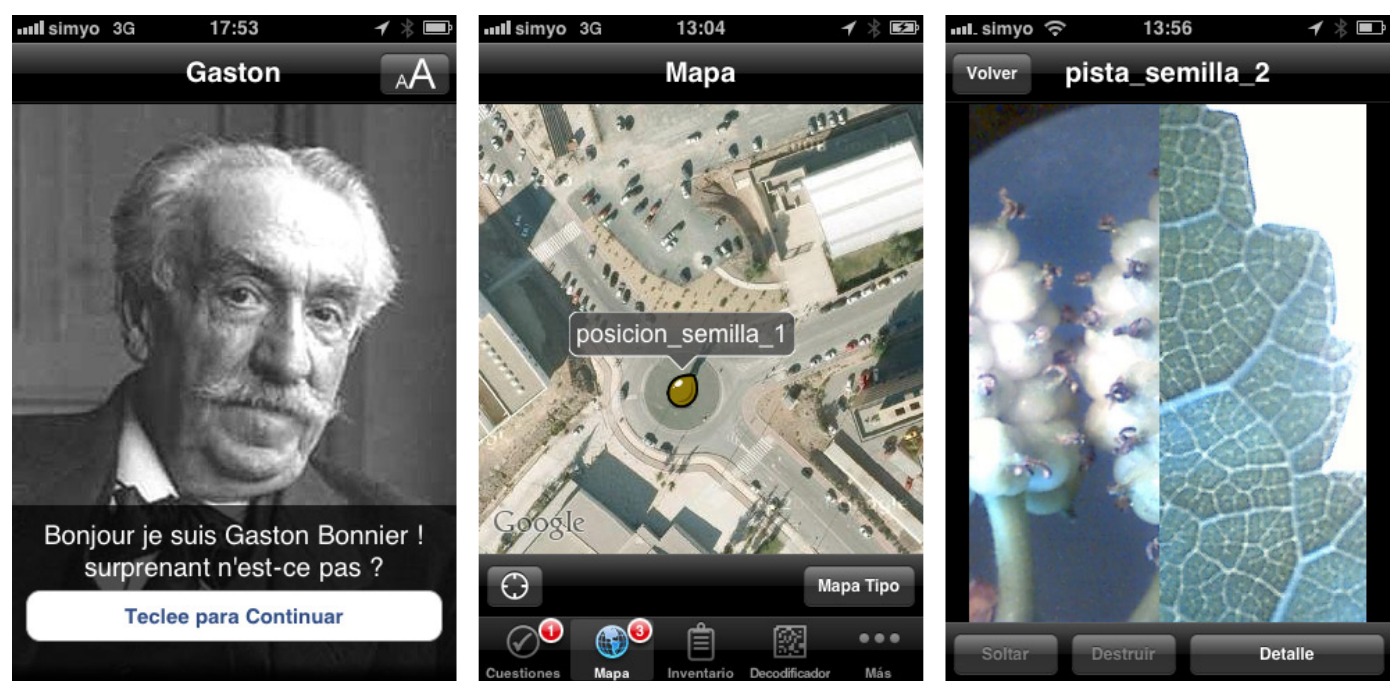

Fig.2.

In this case, contrary to general assumptions, smartphone use is positive for student training. We cannot constrain the applications of these devices, nothing can prevent the student instead of following the proposal, start tweeting, but we can begin a series of positive practices dismantle budgets for both students and teachers. Fred Adam, a professor at the University of Murcia and as autonomous, the Finnish company contacted Keeploop, producing these monocular adaptable phones. The company proposed the creation of an application called Keeploop Challenges and partially extrapolated Microcosmos proposal Explorer. The idea is to create a community that creates challenges linked to the exploration of the microcosm. You can publish photographic images with their corresponding coordinates and challenge other users to locate the source or origin of the image macro. Currently Keeploop Challenges funding is under. This can be seen as an example of how ARIS Games may serve for the development of prototype (as the company Keeploop potential understood thanks to the idea Aris prototype Microcosm Explorer) which subsequently transcends professional development.

\section{CONCLUSIONS}

They focus on strategy on online collaboration and developing a sense of learning community by careful matching of partners, reducing the group size and offering clear and strict requirements for communications. Other researchers such as Linowes, Mroczkowski, Uchida, and Komatsu (2000) introduce the associative group analysis (AGA) derived from linguistic analysis to develop visual portraits that map cultural differences between groups. Maybe it will be a good point to integrate into future perspectives and to be investigated the areas of the e-assessment of the quality of the mobile learning experience. In particular, according with the objectives of this study, we have to consider which types of benefits of mobile learning are provided (chances to access to the learning contents anytime and anywhere).

A particular focus for further research have also shed a light on the user feeling of the different mobile devices as learning tools( how they use them to learn and improve their learning), and last but not least, how they interact with the digital stimuli the new learning environment provide. 
In conclusion, we tried to increase social presence and interaction online and reduce the anxiety and barriers, exploiting the use of social media as learning applications such as Facebook, whatsupps and IPad for cross-cultural collaboration we co-create with the students. Starting from the explicit comprehension of learning styles of the students, trough the interactive workshop we'll try to designing together choices or options to meet the different expectations and learning needs. Developing interactive workshops in a blended environment, we based our focus on the universal design principles (e.g., providing real-time text captioning for all audio, video, and multi-media presentations) to provide access and chances for all (included students with less opportunities background and socio-cognitive problems).

The results obtained from the analysis of the data from the various activities (blended) had the objectives to identify the process where the action should be interactive in the 4th dimensions we described, in order to improve the users' engagement and satisfaction of the training process as a new explorative adventure of dynamic learning. Regarding the future of tools on mobile devices, according to our initial questions, we need to research more about the time gaps in which the mobile devices are used as learning contents providers.

Students, teachers, families, communities and practitioners should develop together with researchers the competences their learning needs to carry out in online environment. Our proposal is providing as a continuum, the general assessment of the interactive workshop where students feedbacks, co-evaluate with the teachers the assignment in the same moment they are doing the learning activities.

Enabling interoperability with OER and encouraging also private-public partnership to fund their projects and give changes to education for all, we discover at the end of the workshop on the five-point Likert scale they indicate that the main problems they had found are regarding the development of the games as module offered. So we consider more important due to the blended nature of the activities, have a first face to face meeting with the students in order to make familiar methodology, devices and create more common and shared goals. From the evaluation of the online questionnaire, we argue that students aim to introduce this kind of activities into daily classroom. In light of these elements, it was thought that on students and teachers' preferences or affordances (Gibson, 1997), in specific blended domain, interactive workshop technique allow us to explore also the importance of new semantic of communication in a digital world, purchase to use m-learning devices to learn and in a more open and shared way, according with the needs they have into a social learning environment, where youngsters shaping their future, based on a cross-cultural understanding, helping us as teacher/facilitator/tutor to foster a meaningful environment is such a changing and fast world. 


\section{BIBLIOGRAPHY}

Jean J. Schensul, LeCompte M., (1999). Essential ethnographic methods: observations, interviews, and questionnaires. Rowman Altamira, Jan 1, 1999.

Chan, S., (2006). mLearning for workplace apprentices: a report on trial is undertaken to establish learning portfolios. Paper presented M-Learn 2006 conference.

Moreno, I. "Narrativa Hipermedia y Transmedia", en Perales Blanco, V. (ed): Creatividad y Discursos Hipermedia, 2012, pp. 21-40.

Gibson, J.C. (1996), The definition, understanding and design of aircraft handling qualities. Agard Conf.Proc.Cp. 